\title{
Comparative Proteomic Analysis for a Putative Pyridoxal Phosphate-Depen- dent Aminotransferase Required for Virulence in Acidovorax citrulli
}

\author{
Jongchan Lee, Lynn Heo, and Sang-Wook Han (D) * \\ Department of Plant Science and Technology, Chung-Ang University, Anseong 17546, Korea \\ (Received on September 9, 2021; Revised on October 6, 2021; Accepted on October 13, 2021)
}

Acidovorax citrulli $(A c)$ is the causative agent of bacterial fruit blotch disease in watermelon. Since resistant cultivars have not yet been developed, the virulence factors/mechanisms of $A c$ need to be characterized. This study reports the functions of a putative pyridoxal phosphate-dependent aminotransferase (PpdaAc) that transfers amino groups to its substrates and uses pyridoxal phosphate as a coenzyme. It was observed that a ppdaAc knockout mutant had a significantly reduced virulence in watermelon when introduced via germinated-seed inoculation as well as leaf infiltration. Comparative proteomic analysis predicted the cellular mechanisms related to PpdaAc. Apart from causing virulence, the PpdaAc may have significant roles in energy production, cell membrane, motility, chemotaxis, post-translational modifications, and iron-related mechanisms. Therefore, it is postulated that PpdaAc may possess pleiotropic effects. These results provide new insights into the functions of a previously unidentified PpdaAc in $A c$.

Keywords : Acidovorax citrulli, proteomics, pyridoxal phosphate-dependent aminotransferase

\footnotetext{
*Corresponding author.

Phone) +82-31-670-3150, FAX) +82-2-670-8845

E-mail)swhan@cau.ac.kr

ORCID

Sang-Wook Han

https://orcid.org/0000-0002-0893-1438
}

Handling Editor : Chang-Jin Park

(c) This is an Open Access article distributed under the terms of the Creative Commons Attribution Non-Commercial License (http:// creativecommons.org/licenses/by-nc/4.0) which permits unrestricted noncommercial use, distribution, and reproduction in any medium, provided the original work is properly cited.

Articles can be freely viewed online at www.ppjonline.org.
Acidovorax citrulli $(A c)$ is a Gram-negative, plant pathogenic bacterium that causes bacterial fruit blotch (BFB) on cucurbit crops, leading to serious crop losses and a subsequent damage to the cucurbit industry (Bahar and Burdman, 2010). Since $A c$ is a seed-borne pathogen, the disease cycle of BFB begins with infected seeds (Song et al., 2020). It is mainly spread by rain splash; the dispersed $A c$ can enter plants through open stomata or through stigmas, thereby leading to a disease outbreak (Johnson et al., 2011). The symptoms of BFB include a water-soaked lesion on the cotyledons and wilting in the initial stage (Burdman and Walcott, 2012). Furthermore, in the infected fruits, watersoaked lesions appear on the fruit surface, finally leading to necrosis of the internal fruit parts. However, in spite of the importance of developing cultivars resistant to $A c$ infection, it has not yet been identified.

Several virulence factors of $A c$ and their related mechanisms have been characterized in previous studies. Similar to other plant pathogenic bacteria, the type II and III secretion systems of $A c$ are crucial for its virulence (Johnson et al., 2015; Zhang et al., 2018). Moreover, the type IV pilimediated motility and quorum-sensing are required for its virulence and seed-transmission (Bahar et al., 2009; Johnson and Walcott, 2013). Recently, two proteins, bifunctional chorismate mutase/prephenate dehydratase (CmpAc) and glycerol-3-phosphate dehydrogenase (GlpdAc), have been identified in Ac (Kim et al., 2020b, 2021). Both proteins are involved in its virulence as well as in other mechanisms, such as twitching motility, tolerance against external stresses, or growth in diverse carbon sources. This suggests that CmpAc and GlpdAc display pleiotropic effects in $A c$. However, other virulence factors of $A c$ and their related mechanisms are poorly understood.

Aminotransferases form a group of enzymes that can remove the amino group from amino acids, transfer it to $\alpha$-keto acids, and thereby produce new amino acid products (Shin et al., 2018). Specific aminotransferases have been 
identified for all amino acids, except lysine, threonine, proline, and hydroxyproline (Ndrepepa and Kastrati, 2019). Apart from regulating amino acid metabolism, aminotransferases are mainly involved in the tricarboxylic acid (TCA) cycle. Some aminotransferases, namely the pyridoxal phosphate-dependent aminotransferases (Ppda), require pyridoxal phosphates as coenzymes for their enzymatic activity (Hafkenscheid and Dijt, 1979). In Mycobacterium tuberculosis, aminotransferases are required for growth and virulence in mice (Jansen et al., 2020). Moreover, the aminotransferases of Xanthomonas oryzae pv. oryzicola, which causes the bacterial leaf streak disease in rice, are also involved in virulence (Guo et al., 2012). However, the role of Ppda in $A c$ has not yet been reported.

We performed two different virulence tests to determine the involvement of pyridoxal phosphate-dependent aminotransferase in $A c$ (PpdaAc) in its virulence. Additionally, the biological and cellular mechanisms related to PpdaAc were predicted using comparative proteomic analysis and clusters of orthologous groups (COG) classification. To identify the genes that are associated with virulence in $A c$, we screened the Tn5-insertional mutant library, as described previously (Kim et al., 2020b). We identified a virulencedeficient mutant in which a putative pyridoxal phosphatedependent aminotransferase gene (ppdaAc) was disrupted by Tn5, according to the manufacturer's protocol (Lucigen, Middleton, WI, USA), and we named it Ac $\triangle p p d a A c$. To generate the complemented strain, the open reading frame of ppdaAc was amplified using a specific primer set (5'-GGTACCATGAAGACGATCCACAAGTC-3' and 5'-CGGGCGGCTGCCGAGCACCACCACCACCA CCACTGAGAGCTC-3') and cloned into pGem-T easy vector (Promega, Madison, WI, USA); the gene sequence was confirmed using Sanger sequencing. The confirmed gene was cleaved by $K p n \mathrm{I}$ and $S a c \mathrm{I}$ and ligated into the broad-host-range vector pBBR1-MCS5, thereby creating the recombinant construct pMCS5-ppdaAc. Thereafter, the construct was introduced into the wild-type $A c$, generating $A c \Delta p p d a A c(\mathrm{PpdaAc})$. To minimize the side effects of vector insertion, an empty vector was introduced into the wild-type and $A c \triangle p p d a A c$ strains, creating $A c(\mathrm{EV})$ and $A c \triangle p p d a A c(\mathrm{EV})$, respectively. All the plasmids and bacterial strains used in this study are listed in Table 1.

To test the pathogenicity, we performed both germinated-seed inoculation and leaf infiltration, as described previously (Kim et al., 2020b). In case of germinated-seed inoculation, all the seedlings infected by $A c(\mathrm{EV})$ were completely wilted, whereas in case of $A c \Delta p p d a A c(\mathrm{EV})$ inoculation, the disease symptoms were absent (Fig. 1A and B). The virulence of the complemented strain was restored to levels similar to that of $A c(\mathrm{EV})$. At 7 days post inoculation, the disease indexes of $A c(\mathrm{EV}), A c \Delta p p d a A c(\mathrm{EV})$, and $A c \triangle p p d a A c($ PpdaAc) reached 2, 0, and 1.6, respectively. Incidentally, the results obtained from the leaf infiltration experiment were similar to those obtained from the germinated-seed inoculation (Fig. 1C and D). The population of

Table 1. Bacterial strains and plasmids used in this study

\begin{tabular}{|c|c|c|}
\hline Strains or plasmids & Characteristic(s) & Source or reference \\
\hline \multicolumn{3}{|l|}{ Escherichia coli } \\
\hline EC100D & For rescuing Tn5-insertional plasmids & Epicentre \\
\hline DH5 $\alpha$ & For cloning & Promega \\
\hline \multicolumn{3}{|l|}{ Acidovorax citurulli } \\
\hline KACC17005 & Wild-type, complete whole genome sequence, Rif ${ }^{r}$ & Kim et al. (2020b) \\
\hline$A c(\mathrm{EV})$ & Wild-type carrying pBBR1-MCS5, Rif, $\mathrm{Gm}^{\mathrm{r}}$ & This study \\
\hline$A c \Delta p p d a A c$ & Knockout mutant, Tn5 inserted in $p p d a A c, \operatorname{Rif}^{\mathrm{r}}, \mathrm{Kan}^{\mathrm{r}}$ & This study \\
\hline$A c \Delta p p d a A c(\mathrm{EV})$ & $A c \Delta p p d a A c$ carrying pBBR1-MCS5, Rif $^{\mathrm{r}}, \mathrm{Kan}^{\mathrm{r}}, \mathrm{Gm}^{\mathrm{r}}$ & This study \\
\hline$A c \Delta p p d a A c(\mathrm{PpdaAc})$ & $A c \triangle p p d a A c$ carrying pMCS5-ppdaAc, $\mathrm{Rif}^{\mathrm{r}}, \mathrm{Kan}^{\mathrm{r}}, \mathrm{Gm}^{\mathrm{r}}$ & This study \\
\hline \multicolumn{3}{|l|}{ Plasmids } \\
\hline pGem-T easy & TA vector, $\mathrm{Amp}^{\mathrm{r}}$ & Promega \\
\hline pGem-ppdaAc & $\begin{array}{l}\text { pGem-T easy containing the open reading frame of ppdaAc }(1,269 \mathrm{bp}) \text {, } \\
\text { Amp }^{\mathrm{r}}\end{array}$ & This study \\
\hline pBBR1-MCS5 & Broad-host-range vector, LacZ promoter, $\mathrm{Gm}^{\mathrm{r}}$ & Kovach et al. (1995) \\
\hline pMCS5-ppdaAc & pBBR1-MCS5 carrying the $p p d a A c$ from $\mathrm{pGem}-p p d a A c, \mathrm{Gm}^{\mathrm{r}}$ & This study \\
\hline
\end{tabular}

Rif $^{r}, \mathrm{Kan}^{\mathrm{r}}, \mathrm{Gm}^{\mathrm{r}}$, and $\mathrm{Amp}^{\mathrm{r}}$ represent resistance to Rifampicin, Kanamycin, Gentamycin, and Ampicillin, respectively. 

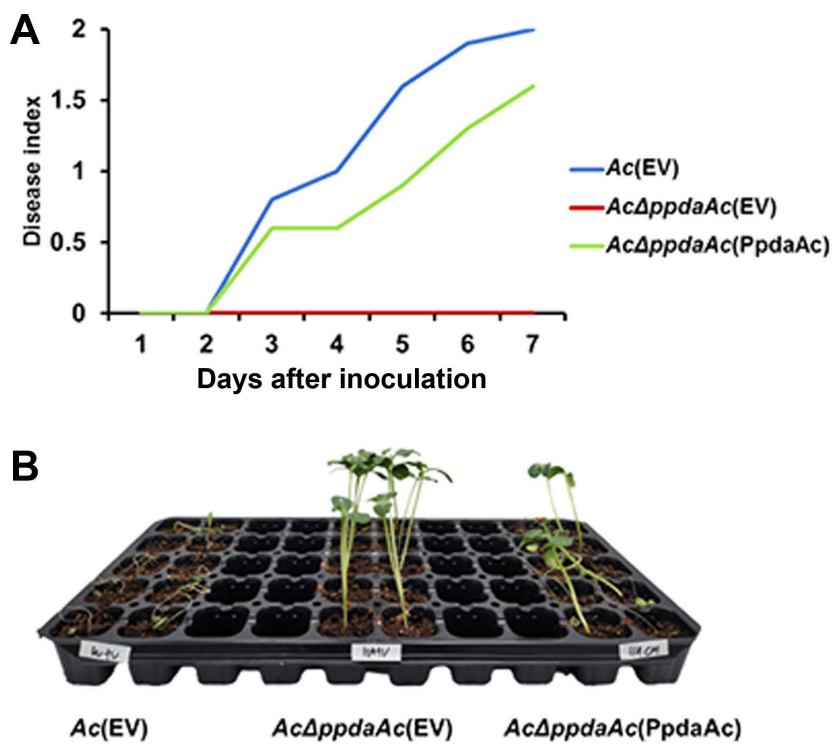

C
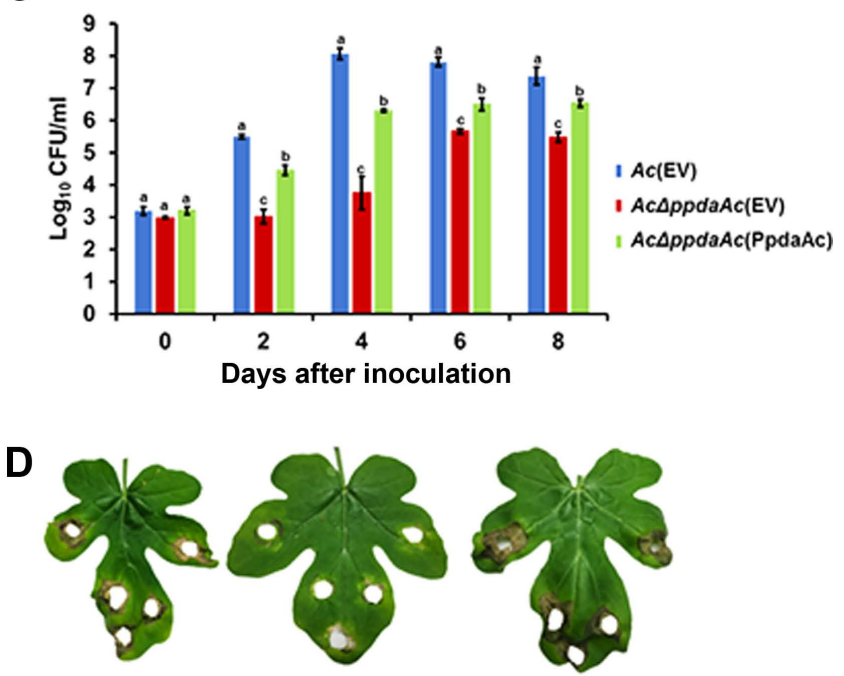

$\boldsymbol{A c}(\mathrm{EV}) \quad \operatorname{Ac} \Delta \mathrm{ppdaAc(EV)} A c \Delta p p d a A c(\mathrm{PpdaAc})$

Fig. 1. Pathogenicity assay for $A c(\mathrm{EV}), A c \Delta p p d a A c(\mathrm{EV})$, and $A c \triangle p p d a A c(\mathrm{PpdaAc})$ using germinated-seed inoculation and leaf infiltration methods in watermelon. (A) The disease index of watermelon seedlings infected by three strains through germinatedseed inoculation till 7 days after inoculation. Disease index $=$ (Number of no symptom seedlings $\times 0+$ numbers of spotted seedlings $\times 1+$ numbers of wilted seedlings $\times 2$ )/Total number of seedlings. (B) A photograph from the germinated-seed inoculation captured $7 \mathrm{~d}$ after inoculation. (C) The infiltrated watermelon true leaf of each strain. The three strains were suspended in 10 $\mathrm{mM} \mathrm{MgCl}$, adjusted to $10^{5} \mathrm{cfu} / \mathrm{ml}$, and inoculated into first and second true leaves of 2-week-old seedlings, using needless syringes. In the infiltration assay, three biological replicates with three technical replicates were performed per strain. The error bars indicate standard deviation, and the different characters on the error bar indicate statistical difference by ANOVA $(P<0.05)$. (D) A photograph from the leaf infiltration captured $6 \mathrm{~d}$ after inoculation.
$A c \triangle p p d a A c(\mathrm{EV})$ was significantly lower than those of the others in the entire period. Leaves infiltrated by $A c(\mathrm{EV})$ and $A c \Delta p p d a A c($ PpdaAc) exhibited dark colored blight symptoms, while those infiltrated by $A c \Delta p p d a A c(\mathrm{EV})$ remained green. Therefore, it seemed that the virulence of Ac $\triangle p p d a A c($ PpdaAc) had been partially complemented. This partial complementation may be attributed to the different promoters and/or copy numbers of the gene in $A c \triangle p p d a A c(\mathrm{PpdaAc})$ because pBRR1-MCS5 maintains several copy numbers in bacteria, and the expression of the targeted gene is driven by the lac $Z$ promoter, which is constitutively expressed (Kovach et al., 1995). Hence, these data demonstrate that PpdaAc is indispensable for virulence in $A c$, which is in accordance with previous studies that have established the involvement of Ppda in virulence of other pathogens. For instance, in the Ppda deletion mutant of Ralstonia solanacearum the virulence of the pathogen is attenuated (Kai et al., 2016). Similarly, in Streptococcus pneumoniae, Ppda is important for the biosynthesis of cell wall (Han et al., 2018), thereby indicating that Ppda is one of its virulence factors.

It is well-known that one protein is involved in virulence and other distinct mechanisms in animal and plant pathogenic bacteria (Felgner et al., 2016; Kim et al., 2020b; Park et al., 2021). Therefore, we aimed to determine PpdaAc-related mechanisms by comparing the abundance of different proteins in $A c$ and $A c \Delta p p d a A c$ strains. Sample preparation, protein extraction, peptide generation, liquid chromatography with tandem mass spectrometry, and protein identification/quantification were performed based upon previously established protocols (Kim et al., 2020b). A total of 896 and 947 proteins were commonly detected in the three biological replicates of the wild-type and the mutant, respectively (Supplementary Table 1). Subsequently, these proteins were subjected to a comparative analysis, the results of which showed that 66 and 115 proteins were exclusively present in $A c$ and $A c \Delta p p d a A c$, respectively; on the contrary, with respect to shared proteins, 35 and 68 proteins were more abundant ( $>2$-fold) in $A c$ and $A c \Delta p p d a A c$, respectively (Fig. 2A). Incidentally, PpdaAc (ATG95109) was detected only in the wild-type and not in the mutant, thereby indicating that the proteomic analysis had been performed successfully. Thereafter, these differentially abundant proteins were classified (Fig. 2B, Supplementary Tables 2 and 3) using the COG categorization (Tatusov et al., 2000). The proteins belonging to group $\mathrm{C}$ (energy production/conversion), E (amino acid transport/ metabolism), G (carbohydrate transport/metabolism), $\mathrm{H}$ (coenzyme transport/metabolism), I (lipid transport/metabolism), N (cell motility), Q (secondary metabolite bio- 
A

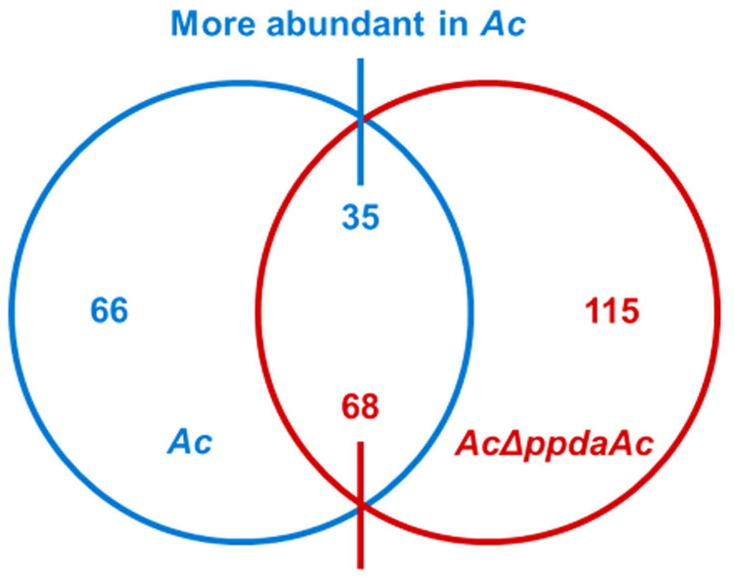

More abundant in AcDppdaAc

B

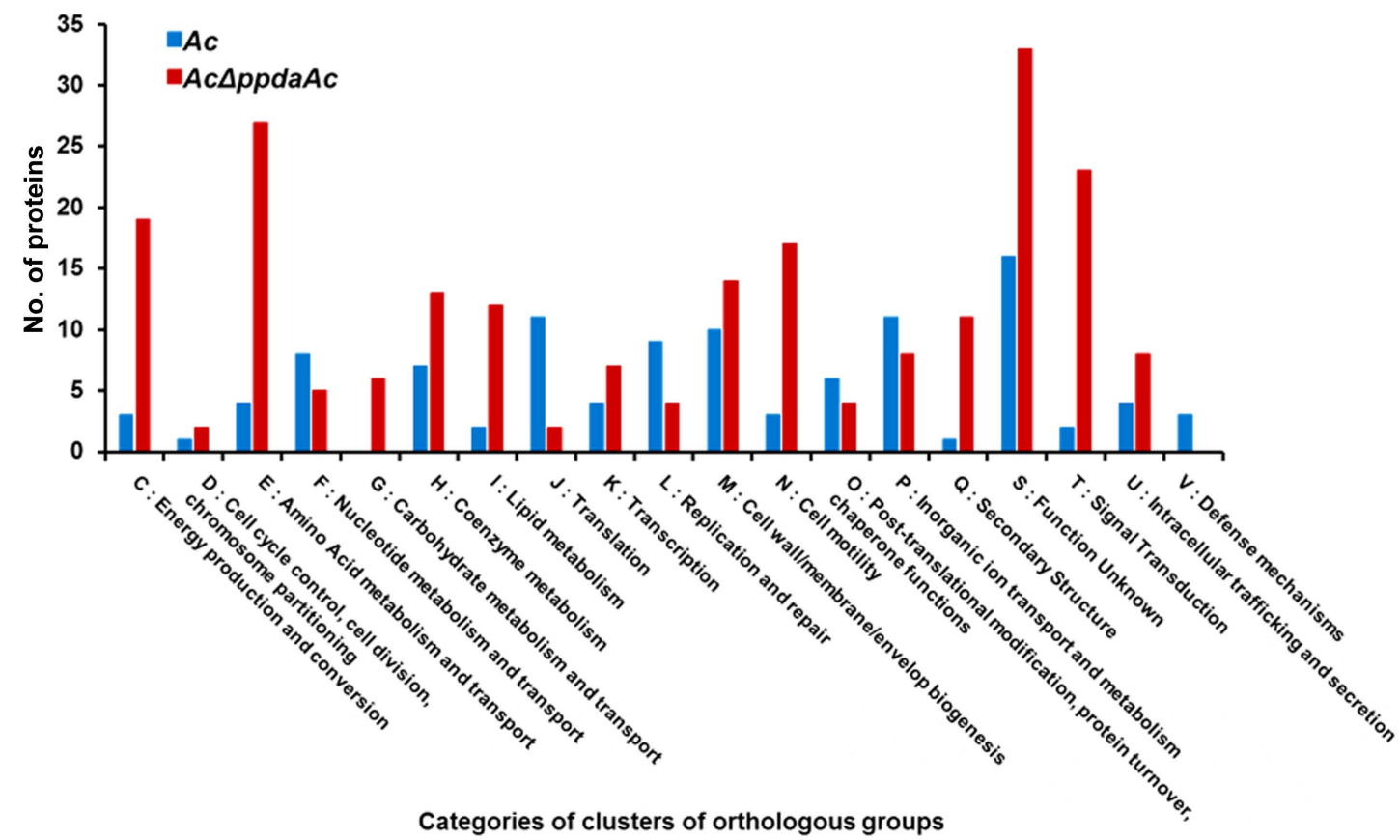

Fig. 2. Comparative proteomic analysis between the wild-type $(A c)$ and the mutant (Ac $\triangle p p d a A c)$. (A) The Venn diagram indicates that 66 and 115 proteins were exclusively detected, and 35 and 68 proteins were more abundant ( $>2$-fold) in $A c$ and $A c \Delta p p d a A c$, respectively. (B) The categories of clusters of orthologous groups for the differentially abundant proteins ( $>2$-fold) from the comparative analysis.

synthesis/transport/catabolism), and $\mathrm{T}$ (signal transduction) were highly abundant in the mutant, whereas, the proteins categorized in group F (nucleotide transport/metabolism), $\mathrm{J}$ (translation), L (DNA replication/recombination/repair), $\mathrm{O}$ (post-translational modification, protein turnover, and chaperones), and $\mathrm{P}$ (inorganic ion transport/metabolism) were more abundant in the wild-type. The representative proteins and their respective predicted mechanisms, based upon the proteomic analysis, are described in Fig. 3.

Living organisms acquire energy through diverse en- ergy production pathways, including glycolysis (group G), TCA cycle (group E), and electron transport system (group C) (Fernie et al., 2004). The results of the proteomic analysis showed that the mutant strain expressed the proteins included in these groups more abundantly, as compared to the respective protein expressions in the wildtype. Moreover, gluconeogenesis-related proteins, namely glucose-6-phosphate isomerase (ATG97016) and fructose 1, 6-bisphosphatase (ATG94560), were more abundant in the mutant than in the wild-type. Furthermore, isocitrate 
lyase (ATG96777) that is essential in the glyoxylate pathway, an alternative pathway for carbon metabolism (Chew et al., 2019), was more abundant in AcAppdaAc than in the wild-type. Normally, isocitrate is utilized in the TCA cycle; however, when bacteria utilize fatty acids for growth, they use the isocitrate in the glyoxylate pathway for gluconeogenesis (Gonçalves et al., 2016). Since our results show a greater abundance of fatty acid-related proteins in the mutant than in the wild-type, it may be predicted that PpdaAc affects gluconeogenesis from fatty acids.

Previous studies have shown that Ppda is associated with glutamate production (Ndrepepa and Kastrati, 2019). In this study, we observed that glutamate 5-kinase (ATG93720), which is involved in glutamate synthesis, was more abundant in the wild-type than in the mutant. In biochemical pathways, glutamates are converted to aspartates, which, in turn, can be degraded to homoserine by homoserine dehydrogenase; the homoserines can be further converted to methionine and threonine (Kim et al., 2020a). Based upon our data, the enzymes necessary for the abovementioned biochemical pathways, homoserine dehydrogenase (ATG95108), threonine synthase (ATG95107), and methionine synthase (ATG95998), are more abundant in the wild-type than in the mutant. Therefore, it can be postulated that glutamate synthesis would be significantly re- duced in the mutant due to the lack of PpdaAc expression. This may lead to the increased expression of other enzymes responsible for glutamate production, such as glutamine amidotransferase, in the mutant. Incidentally, glutamine amidotransferase (ATG95676), which is involved in glutamate production, was more abundant in $A c \Delta p p d a A c$ than in the wild-type. Electron transfer system has four oxidoreductase complexes, namely NADH dehydrogenase (complex I), succinate dehydrogenase (complex II), cytochrome c reductase (complex III), and cytochrome c oxidase (complex IV) (Schertl and Braun, 2014). In the proteomic analysis, we detected the presence of succinate dehydrogenase (ATG93227), NADH-quinone oxidoreductase subunit (ATG96994), flavoprotein subunit alpha (ATG93258), and glycerol-3-phosphate dehydrogenase/oxidase (ATG95651), which are associated with complex I and II (Fig. 3), and cytochrome c oxidase subunit II (ATG93913), cytochrome C4 (ATG93956), and 2Fe-2S ferredoxin (ATG95897), which are associated with complex III and IV. These results indicate that PpdaAc is related to amino acid metabolism as well as electron/energy production.

Since the abundance of the diverse proteins categorized in cell motility was altered (Fig. 2B), it is possible that PpdaAc is involved in bacterial motility; for example, flagella component proteins (ATG96515 and ATG96513) and pili-

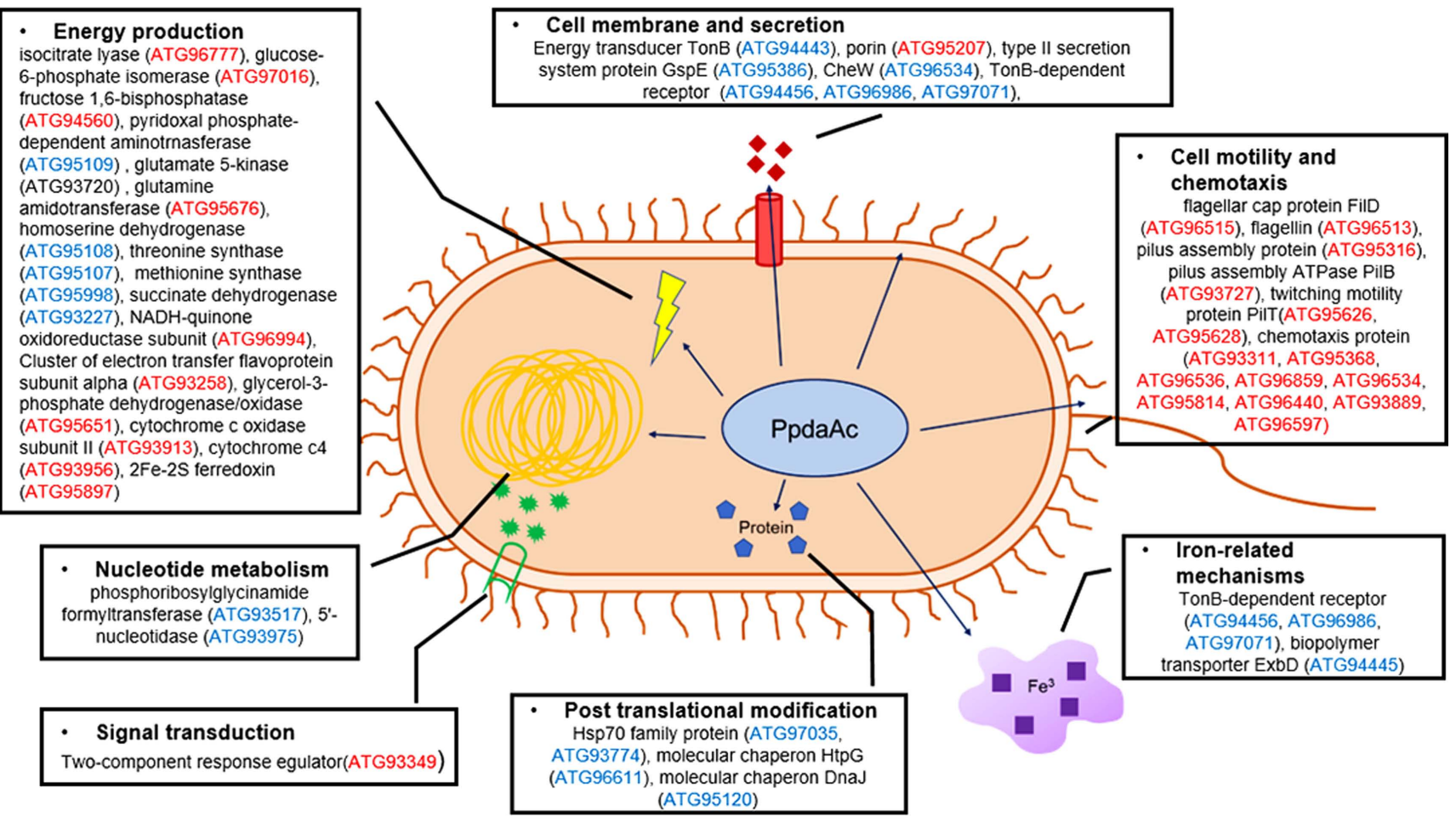

Fig. 3. A schematic diagram for putative biological and cellular mechanisms related to PpdaAc in Acidovorax citrulli. Blue and red accession numbers indicate proteins more abundant in the wild-type $(A c)$ and the mutant $(A c \triangle p p d a A c)$, respectively. 
related proteins (ATG95316, ATG93727, ATG95626, and ATG95628) were more abundant in the mutant than in the wild-type (Supplementary Tables 2 and 3). Both flagellaand pili-dependent motilities are tightly associated with virulence in Gram-negative bacteria including Ac, Salmonella enterica, and Pseudomonas aeruginosa (Bahar et al., 2009; Giltner et al., 2010; Kim et al., 2020b; Yang et al., 2012). However, virulence decreases when a flagella-related gene is overexpressed (Yang et al., 2012). Chemotaxis-related proteins (ATG93311, ATG95368, ATG96536, ATG96859, ATG96534, ATG95814, ATG96440, ATG93889, and ATG96597) were also identified in the comparative proteomic analysis. Chemotaxis is the bacterial movement towards particular environmental cues using the motility functions (Wadhams and Armitage, 2004). The CheW is a crucial protein with respect to chemotactic movement in $E$. coli and Rhodobacter sphaeroides (Hamblin et al., 1997; Sanders et al., 1989). Based upon our results, the CheW (ATG96534) protein was more abundant in the wild-type. Hence, it may be inferred that PpdaAc affects the regulation of motility-related proteins, which, in turn, may contribute to virulence in $A c$.

In the proteomic analysis, we detected the presence of proteins of the heat shock protein 70 family (ATG97035 and ATG93774) as well as molecular chaperones DnaJ (ATG95120) and HtpG (ATG96611) (Fig. 3). These proteins are associated with protection against stress conditions and virulence in pathogenic bacteria (Cui et al., 2017; De Maio, 1999; King et al., 2014). Additionally, several proteins related to inorganic ion transport/metabolism were also identified, especially diverse TonB-dependent receptors (ATG94456, ATG96986, and ATG97071) in the wild-type; these receptors are used for obtaining iron via siderophores (Fujita et al., 2019). In Acinetobacter baumannii and Pseudomonas fluorescens, mutation of TonBdependent receptors triggers reduced biofilm formation, adherence, and virulence (Abdollahi et al., 2018; Hu et al., 2012). The biopolymer transporter ExbD (ATG94445) that can form a complex with TonB (TonB-ExbD complex) and transport the iron-siderophore into the cytoplasm through the cell membrane using proton motive forces (Ollis and Postle, 2012) was also detected. The exbD-knockout mutant of Pasteurella multocida shows reduced virulence (Bosch et al., 2002). However, a two-component system response regulator (ATG93349) was only identified in the comparative proteomic analysis, indicating that PpdaAc may not have a significant role in the signaling pathway. Therefore, our results suggest that PpdaAc has a role in post-translation modifications and ion transport but not in the signal transduction system.
In this study, we performed two different pathogenicity tests and demonstrated that PpdaAc is essential for the virulence of $A c$. To determine the biological and cellular mechanisms associated with the PpdaAc protein, we compared the patterns of protein expression between the wild-type and the mutant using proteomic analysis. The comparative analysis revealed that PpdaAc is associated with virulence as well as other biological and cellular mechanisms, thereby suggesting that PpdaAc may have pleiotropic effects in Ac. Nevertheless, it is still unclear how the PpdaAc protein is involved in the predicted mechanisms. Hence, further studies need to be performed to elucidate the specific functions of PpdaAc at molecular and phenotypic levels. Those results will provide a new perspective of the mechanisms/ functions related to Ppda in Gram-negative bacteria.

\section{Conflicts of Interest}

No potential conflict of interest relevant to this article was reported.

\section{Acknowledgments}

This work was supported by the National Research Foundation of Korea (NRF) grant funded by the Korean government (MSIT) (No. NRF-2020R1A2C1013040). This research was supported by the Chung-Ang University research grant in 2021. (to S.W. Han).

\section{Electronic Supplementary Material}

Supplementary materials are available at The Plant Pathology Journal website (http://www.ppjonline.org/).

\section{References}

Abdollahi, S., Rasooli, I. and Mousavi Gargari, S. L. 2018. The role of TonB-dependent copper receptor in virulence of Acinetobacter baumannii. Infect. Genet. Evol. 60:181-190.

Bahar, O. and Burdman, S. 2010. Bacterial fruit blotch: a threat to the cucurbit industry. Isr. J. Plant Sci. 58:19-31.

Bahar, O., Goffer, T. and Burdman, S. 2009. Type IV Pili are required for virulence, twitching motility, and biofilm formation of Acidovorax avenae subsp. citrulli. Mol. Plant-Microbe Interact. 22:909-920.

Bosch, M., Garrido, E., Llagostera, M., Pérez de Rozas, A. M., Badiola, I. and Barbé, J. 2002. Pasteurella multocida exbB, exbD and ton $B$ genes are physically linked but independently transcribed. FEMS Microbiol. Lett. 210:201-208.

Burdman, S. and Walcott, R. 2012. Acidovorax citrulli: generating basic and applied knowledge to tackle a global threat to 
the cucurbit industry. Mol. Plant Pathol. 13:805-815.

Chew, S. Y., Chee, W. J. Y. and Than, L. T. L. 2019. The glyoxylate cycle and alternative carbon metabolism as metabolic adaptation strategies of Candida glabrata: perspectives from Candida albicans and Saccharomyces cerevisiae. J. Biomed. Sci. 26:52.

Cui, J., Ma, C., Ye, G., Shi, Y., Xu, W., Zhong, L., Wang, J., Yin, Y., Zhang, X. and Wang, H. 2017. DnaJ (hsp40) of Streptococcus pneumoniae is involved in bacterial virulence and elicits a strong natural immune reaction via PI3K/JNK. Mol. Immunol. 83:137-146.

De Maio, A. 1999. Heat shock proteins: facts, thoughts, and dreams. Shock 11:1-12.

Felgner, S., Frahm, M., Kocijancic, D., Rohde, M., Eckweiler, D., Bielecka, A., Bueno, E., Cava, F., Abraham, W.-R., Curtiss, R., 3rd, Häussler, S., Erhardt, M. and Weiss, S. 2016. aroAdeficient Salmonella enterica serovar typhimurium is more than a metabolically attenuated mutant. Mbio 7:e01220-16.

Fernie, A. R., Carrari, F. and Sweetlove, L. J. 2004. Respiratory metabolism: glycolysis, the TCA cycle and mitochondrial electron transport. Curr. Opin. Plant Biol. 7:254-261.

Fujita, M., Mori, K., Hara, H., Hishiyama, S., Kamimura, N. and Masai, E. 2019. A TonB-dependent receptor constitutes the outer membrane transport system for a lignin-derived aromatic compound. Commun. Biol. 2:432.

Giltner, C. L., Habash, M. and Burrows, L. L. 2010. Pseudomonas aeruginosa minor pilins are incorporated into type IV pili. J. Mol. Biol. 398:444-461.

Gonçalves, I. L., Mielniczki-Pereira, A. A., Piovezan Borges, A. C. and Valduga, A. T. 2016. Metabolic modeling and comparative biochemistry in glyoxylate cycle. Acta Sci. Biol. Sci. 38:1-6.

Guo, W., Cui, Y.-P., Li, Y.-R., Che, Y.-Z., Yuan, L., Zou, L.-F., Zou, H.-S. and Chen, G.-Y. 2012. Identification of seven Xanthomonas oryzae pv. oryzicola genes potentially involved in pathogenesis in rice. Microbiology 158:505-518.

Hafkenscheid, J. C. M. and Dijt, C. C. M. 1979. Determination of serum aminotransferases: activation by pyridoxal-5'phosphate in relation to substrate concentration. Clin. Chem. 25:55-59.

Hamblin, P. A., Bourne, N. A. and Armitage, J. P. 1997. Characterization of the chemotaxis protein CheW from Rhodobacter sphaeroides and its effect on the behaviour of Escherichia coli. Mol. Microbiol. 24:41-51.

Han, X., Sun, R., Sandalova, T. and Achour, A. 2018. Structural and functional studies of Spr1654: an essential aminotransferase in teichoic acid biosynthesis in Streptococcus pneumoniae. Open Biol. 8:170248.

Hu, Y.-H., Dang, W. and Sun, L. 2012. A TonB-dependent outer membrane receptor of Pseudomonas fluorescens: virulence and vaccine potential. Arch. Microbiol. 194:795-802.

Jansen, R. S., Mandyoli, L., Hughes, R., Wakabayashi, S., Pinkham, J. T., Selbach, B., Guinn, K. M., Rubin, E. J., Sacchettini, J. C. and Rhee, K. Y. 2020. Aspartate aminotransfer- ase Rv3722c governs aspartate-dependent nitrogen metabolism in Mycobacterium tuberculosis. Nat. Commun. 11:1960.

Johnson, K. L. and Walcott, R. R. 2013. Quorum sensing contributes to seed-to-seedling transmission of Acidovorax citrulli on watermelon. J. Phytopathol. 161:562-573.

Johnson, K. L., Minsavage, G. V., Le, T., Jones, J. B. and Walcott, R. R. 2011. Efficacy of a nonpathogenic Acidovorax citrulli strain as a biocontrol seed treatment for bacterial fruit blotch of cucurbits. Plant Dis. 95:697-704.

Johnson, T. L., Waack, U., Smith, S., Mobley, H. and Sandkvist, M. 2015. Acinetobacter baumannii is dependent on the type II secretion system and its substrate LipA for lipid utilization and in vivo fitness. J. Bacteriol. 198:711-719.

Kai, K., Ohnishi, H., Kiba, A., Ohnishi, K. and Hikichi, Y. 2016. Studies on the biosynthesis of ralfuranones in Ralstonia solanacearum. Biosci. Biotechnol. Biochem. 80:440-444.

Kim, D. H., Nguyen, Q. T. and Yang, J. K. 2020a. Biochemical characterization of homoserine dehydrogenase from Pseudomonas aeruginosa. Bull. Korean Chem. Soc. 41:127-132.

Kim, M., Lee, J., Heo, L. and Han, S.-W. 2020b. Putative bifunctional chorismate mutase/prephenate dehydratase contributes to the virulence of Acidovorax citrulli. Front. Plant Sci. 11:569552.

Kim, M., Lee, J., Heo, L., Lee, S. J. and Han, S.-W. 2021. Proteomic and phenotypic analyses of a putative glycerol3-phosphate dehydrogenase required for virulence in Acidovorax citrulli. Plant Pathol. J. 37:36-46.

King, A. M., Pretre, G., Bartpho, T., Sermswan, R. W., Toma, C., Suzuki, T., Eshghi, A., Picardeau, M., Adler, B. and Murray, G. L. 2014. High-temperature protein $\mathrm{G}$ is an essential virulence factor of Leptospira interrogans. Infect. Immun. 82:11231131.

Kovach, M. E., Elzer, P. H., Hill, D. S., Robertson, G. T., Farris, M. A., Roop, R. M. 2nd. and Peterson, K. M. 1995. Four new derivatives of the broad-host-range cloning vector $\mathrm{pB}$ BR1MCS, carrying different antibiotic-resistance cassettes. Gene 166:175-176.

Ndrepepa, G. and Kastrati, A. 2019. Alanine aminotransferase-a marker of cardiovascular risk at high and low activity levels. $J$. Lab. Precis. Med. 4:29.

Ollis, A. A. and Postle, K. 2012. Identification of functionally important TonB-ExbD periplasmic domain interactions in vivo. J. Bacteriol. 194:3078-3087.

Park, H.-J., Seong, H. J., Lee, J., Heo, L., Sul, W. J. and Han, S.W. 2021. Two DNA methyltransferases for site-Specific $6 \mathrm{~mA}$ and $5 \mathrm{mC}$ DNA modification in Xanthomonas euvesicatoria. Front. Plant Sci. 12:621466.

Sanders, D. A., Mendez, B. and Koshland, D. E. Jr. 1989. Role of the CheW protein in bacterial chemotaxis: overexpression is equivalent to absence. J. Bacteriol. 171:6271-6278.

Schertl, P. and Braun, H.-P. 2014. Respiratory electron transfer pathways in plant mitochondria. Front. Plant Sci. 5:163.

Shin, Y.-C., Yun, H. and Park, H. H. 2018. Structural dynamics of the transaminase active site revealed by the crystal structure 
of a co-factor free omega-transaminase from Vibrio fluvialis JS17. Sci. Rep. 8:11454.

Song, Y.-R., Hwang, I. S. and Oh, C.-S. 2020. Natural variation in virulence of Acidovorax citrulli isolates that cause bacterial fruit blotch in watermelon, depending on infection routes. Plant Pathol. J. 36:29-42.

Tatusov, R. L., Galperin, M. Y., Natale, D. A. and Koonin, E. V. 2000. The COG database: a tool for genome-scale analysis of protein functions and evolution. Nucleic Acids Res. 28:33-36. Wadhams, G. H. and Armitage, J. P. 2004. Making sense of it all: bacterial chemotaxis. Nat. Rev. Mol. Cell Biol. 5:1024-1037. Yang, X., Thornburg, T., Suo, Z., Jun, S., Robison, A., Li, J., Lim, T., Cao, L., Hoyt, T., Avci, R. and Pascual, D. W. 2012. Flagella overexpression attenuates Salmonella pathogenesis. PLoS ONE 7:e46828.

Zhang, X., Zhao, M., Yan, J., Yang, L., Yang, Y., Guan, W., Walcott, R. and Zhao, T. 2018. involvement of $h r p X$ and $h r p G$ in the virulence of Acidovorax citrulli strain Aac5, causal agent of bacterial fruit blotch in cucurbits. Front. Microbiol. 9:507. 